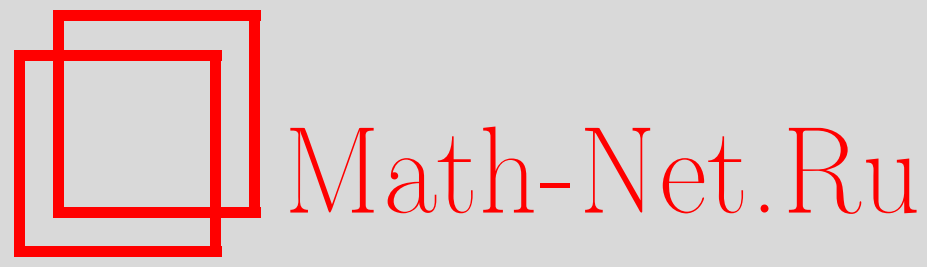

Г. Л. Рыбников, О фундаментальной группе дополнения к комплексной конфигурации гиперплоскостей, Функи. анализ и его прил., 2011, том 45, выпуск $2,71-85$

DOI: https://doi.org/10.4213/faa3025

Использование Общероссийского математического портала MathNet.Ru подразумевает, что вы прочитали и согласны с пользовательским соглашением

http://www . mathnet.ru/rus/agreement

Параметры загрузки:

IP : 54.198 .64 .247

26 апреля 2023 г., 17:04:08

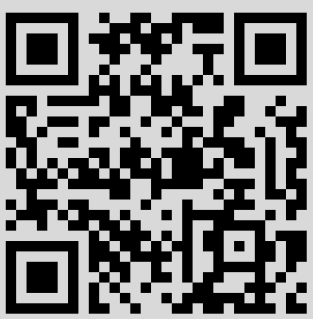




\title{
О фундаментальной группе дополнения к комплексной конфигурации гиперплоскостей*
}

\author{
(c) 2011. Г. Л. РыБников
}

\begin{abstract}
Светлой памяти И. М. Гельфанда
В работе построены две комбинаторно эквивалентные конфигурации прямых на комплексной проективной плоскости, у которых фундаментальные группы дополнений не изоморфны. В доказательстве используется новый инвариант фундаментальной группы дополнения к конфигурации прямых заданного комбинаторного типа относительно изоморфизмов, индуцирующих канонический изоморфизм первых групп гомологий.
\end{abstract}

Эта статья является переработанным вариантом препринта [8], вышедшего в 1994 г. и впоследствии неоднократно цитировавшегося разными авторами. Используемый здесь инвариант позднее обсуждался, в частности, в работе [2]. Я благодарен В. А. Васильеву, побудившему меня представить работу к публикации, и С. А. Юзвинскому, давшему мне ряд советов по улучшению текста.

Я благодарен рецензентам, замечания которых позволили мне исправить ряд недочетов первоначального варианта статьи.

\section{§1. Введение}

Многие топологические свойства комплексной конфигурации гиперплоскостей можно описать в терминах ее комбинаторной структуры (матроида). Например, так обстоят дела для кольца когомологий дополнения к конфигурации [6] и для мальцевского пополнения фундаментальной группы такого дополнения [3]. Однако все известные алгоритмы вычисления самой фундаментальной группы дополнения используют нематроидные данные. (В случае комплексификации вещественной конфигурации ответ может быть дан в терминах ориентированного матроида, что требует больше информации, чем просто размерности всевозможных пересечений гиперплоскостей конфигурации, как для обычного матроида.)

Согласно классической теореме Зарисского, фундаментальная группа дополнения к гиперповерхности в $\mathbb{C} P^{n}$ изоморфна фундаментальной группе дополнения к пересечению этой гиперповерхности с общей двумерной плоскостью в этой плоскости. Поэтому в нашем случае достаточно изучить фундаментальные группы дополнений к конфигурациям прямых в $\mathbb{C} P^{2}$. В настоящей работе мы описываем комбинаторную структуру конфигураций прямых в терминах

*Работа выполнена при поддержке грантов РФФИ №09-01-12185-офи_м и ВШЭ 09-090010 и проекта Т3-62.0 «Математические исследования в области маломерной топологии, алгебраической геометрии, теории представлений» ВШЭ. 
структуры инцидентности, что по сути эквивалентно использованию матроидного языка, но имеет преимущество большей наглядности.

Цель настоящей работы - построить две комбинаторно эквивалентные конфигурации прямых в $\mathbb{C} P^{2}$, фундаментальные группы дополнений к которым неизоморфны. Опишем вкратце наш подход.

Пусть $X$ - дополнение к конфигурации прямых $\mathfrak{L}$, и пусть $C$ - структура инцидентности, которая описывает комбинаторную структуру конфигурации $\mathfrak{L}$. Обозначим через $H$ свободную абелеву группу с базисом, занумерованным прямыми структуры инцидентности $C$. Первая группа гомологий $H_{1}(X, \mathbb{Z})-$ свободная абелева группа с базисом из циклов, обходящих в положительном направлении прямые конфигурации $\mathfrak{L}$. Таким образом, она канонически изоморфна группе $H$. Пусть $X^{\prime}$ - дополнение к еще одной конфигурации прямых, реализующих ту же структуру $C$. В силу канонических изоморфизмов $H_{1}(X, \mathbb{Z}) \cong H$ и $H_{1}\left(X^{\prime}, \mathbb{Z}\right) \cong H$ мы имеем канонические проекции $\pi_{1}(X) \rightarrow H$ и $\pi_{1}\left(X^{\prime}\right) \rightarrow H$, ядрами которых являются коммутанты групп $\pi_{1}(X)$ и $\pi_{1}\left(X^{\prime}\right)$.

Прежде всего, мы получим некоторое необходимое условие для существования изоморфизма $\pi_{1}(X) \rightarrow \pi_{1}\left(X^{\prime}\right)$, согласованного с этими проекциями, т. е. индуцирующего тождественный автоморфизм группы $H$. Для этого по заданной структуре $C$ мы строим некоторую инвариантную конструкцию, зависящую от группы $\pi_{1}(X)$, рассматриваемой вместе с проекцией $\pi_{1}(X) \rightarrow H$. Если эта инвариантная конструкция дает разные результаты для групп $\pi_{1}(X)$ и $\pi_{1}\left(X^{\prime}\right)$, то изоморфизм $\pi_{1}(X) \rightarrow \pi_{1}\left(X^{\prime}\right)$ рассматриваемого типа существовать не может. Построенный инвариант зависит только от $\pi_{1}(X) / \gamma_{4} \pi_{1}(X)$, где $\gamma_{k} G$ есть $k$-й член нижнего центрального ряда группы $G$.

Далее мы рассматриваем две комплексно-сопряженные реализации структуры инцидентности $C_{8}$, соответствующей матроиду Маклейна [4] (см. также [10]), и показываем, что наш инвариант различает эти реализации. Мы также доказываем, что для структуры инцидентности $C_{8}$ группа автоморфизмов группы $H$, получающихся редукцией из автоморфизмов группы $\pi_{1}(X)$, изоморфна группе автоморфизмов самой структуры инцидентности. Наконец, мы комбинируем эти результаты, чтобы показать, что структура инцидентности $C_{13}$, полученная посредством склеивания двух экземпляров структуры Маклейна, имеет по крайней мере две реализации с неизоморфными фундаментальными группами дополнений.

Я глубоко благодарен за обсуждения и советы Т. В. Алексеевской, А. Дрессу, А. Б. Гончарову, С. М. Львовскому, Н. Е. Мневу, Г. А. Носкову, С. Ю. Оревкову, Д. Стоуну, Г. Циглеру и особенно И. М. Гельфанду, без которого эта статья никогда бы не была написана.

\section{§2. Конструкция различающего инварианта}

Определение 1. Структурой инцидентности называется тройка $C=(\mathscr{L}$, $\mathscr{P}, \succ)$, состоящая из множества прямых $\mathscr{L}$, множества точек $\mathscr{P}$ и бинарного соответствия $\succ$, называемого инцидентностью, между $\mathscr{L}$ и $\mathscr{P}$. При этом требуется выполнение следующих аксиом:

(1) для любых двух различных прямых $l, l^{\prime} \in \mathscr{L}$ существует единственная точка $p \in \mathscr{P}$, такая, что $l \succ p$ и $l^{\prime} \succ p$;

(2) любая точка инцидентна по меньшей мере двум прямым. 
Если $l, l^{\prime}$ и $p$ - такие, как в аксиоме (1), то мы пишем $p=l \cap l^{\prime}$ и называем $p$ точкой пересечения прямых $l$ и $l^{\prime}$. Назовем структуру $C=(\mathscr{L}, \mathscr{P}, \succ)$ невырожденной, если $|\mathscr{P}|>1$, т. е. если не все прямые пересекаются в одной точке ${ }^{1)}$.

Определение 2. Комплексной реализаиией структуры инцидентности $C=$ $(\mathscr{L}, \mathscr{P}, \succ)$ называется инъективное отображение $\phi$ из $\mathscr{L}$ в множество прямых на $\mathbb{C} P^{2}$ и из $\mathscr{P}$ в множество точек на $\mathbb{C} P^{2}$, такое, что $\phi p \in \phi l$ тогда и только тогда, когда $p \prec l$.

Пусть $C=(\mathscr{L}, \mathscr{P}, \succ)$ - конечная невырожденная структура инцидентности. Пронумеруем элементы из $\mathscr{L}, \mathscr{L}=\left\{l_{0}, l_{1}, \ldots, l_{n}\right\}$, и назовем $l_{0}$ бесконечно удаленной прямой. Положим $\mathscr{P}_{0}=\left\{p \in \mathscr{P} \mid p \nprec l_{0}\right\}$. Опишем в абстрактных терминах предложенное в [1] описание фундаментальной группы дополнения к конфигурации прямых в $\mathbb{C}^{2}$ в терминах образующих и соотношений (см. также [7]).

Пусть $F-$ свободная группа с образующими $w_{1}, \ldots, w_{n}$, и пусть $\mathscr{A}=\{(i, p) \in$ $\left.\{1, \ldots, n\} \times \mathscr{P}_{0} \mid p \prec l_{i}\right\}$. Предположим, что для каждой пары $(i, p) \in \mathscr{A}$ выбран элемент $w_{i}(p) \in F$, сопряженный в $F$ к $w_{i}: w_{i}(p)=(g(i, p))^{-1} w_{i} g(i, p)$ для некоторого $g(i, p) \in F$. Таким образом, элементы $w_{i}(p)$ задаются некоторым отображением $g: \mathscr{A} \rightarrow F$.

Пусть $p \in \mathscr{P}_{0}$ и $\left\{i_{1}, \ldots, i_{k}\right\}=\left\{i \in\{1, \ldots, n\} \mid l_{i} \succ p\right\}$, причем $i_{1}<\cdots<i_{k}$. Положим $c_{s}(p)=w_{i_{s}}(p) w_{i_{s-1}}(p) \cdots w_{i_{1}}(p) w_{i_{k}}(p) \cdots w_{i_{s+1}}(p)$ для $s=1, \ldots, k$ и $r\left(i_{s}, p\right)=c_{s-1}(p)^{-1} c_{s}(p)$ для $s=1, \ldots, k$, где $c_{0}(p)=c_{k}(p)$. Легко видеть, что $r\left(i_{s}, p\right)=\left[w_{i_{s}}(p), c_{s}(p)\right]$ и $\prod_{s=1}^{k} r\left(i_{s}, p\right)=1$, поэтому лишь $k-1$ из элементов $r\left(i_{s}, p\right)$ независимы. Рассмотрим для каждого $p \in \mathscr{P}_{0}$ множество $\left\{r\left(i_{2}, p\right), \ldots\right.$, $\left.r\left(i_{k}, p\right)\right\}$ независимых элементов, и пусть $\mathscr{R}=\mathscr{R}(g)=\left\{r(i, p) \mid l_{i} \succ p, i \neq \min \{j \mid\right.$ $\left.\left.l_{j} \succ p\right\}\right\}$ - объединение этих множеств. Положим $G=G(\mathscr{R})=F /\langle\mathscr{R}\rangle$, где $\langle\mathscr{R}\rangle$ - минимальная нормальная подгруппа в $F$, содержащая $\mathscr{R}$.

Пусть $\phi$ - комплексная реализация структуры $C$, и пусть $X=\mathbb{C}^{2} \backslash \bigcup_{l \in \mathscr{L}} \phi l$. Тогда, согласно [1], имеет место изоморфизм $\pi_{1}(X) \cong G(\mathscr{R}(g))$ для некоторого выбора отображения $g$. При этом изоморфизме образующая $w_{i}$ соответствует маленькой петле, обходящей $\phi l_{i}$ в положительном направлении (прямые в $\mathbb{C}^{2}$ имеют каноническую коориентацию).

Рассмотрим нижний центральный ряд $\left(\gamma_{k} F\right)$ группы $F\left(\gamma_{1} F=F, \gamma_{k+1} F=\right.$ $\left.\left[F, \gamma_{k} F\right]\right)$. Хорошо известно, что $\operatorname{gr}_{k} F=\gamma_{k} F / \gamma_{k+1} F-$ свободные абелевы группы конечного ранга. Более того, gr $F=\bigoplus_{k=1}^{\infty} \operatorname{gr}_{k} F$ естественно изоморфна (как градуированная абелева группа) свободной алгебре Ли $L$ над $\mathbb{Z}$ с $n$ образующими $x_{1}, \ldots, x_{n}$, являющимися образами элементов $w_{1}, \ldots, w_{n}$ в $F / \gamma_{2} F ; L=$ $\bigoplus_{k=1}^{\infty} L_{k}$ градуирована степенью мономов (см., например, [5, гл. 5]). При этом изоморфизме лиевский коммутатор в $L$ отвечает групповому коммутатору в $F$. Всюду далее мы будем отождествлять $\operatorname{gr}_{k} F$ с $L_{k}$. Положим $H=L_{1}=F / \gamma_{2} F$; это свободная абелева группа с базисом $x_{1}, \ldots, x_{n}$. В случае когда $G=\pi_{1}(X)$ для некоторой комплексной реализации структуры $C$, мы имеем $H \cong H_{1}(X, \mathbb{Z})$, причем $\left(x_{i}\right)$ - это базис, двойственный к базису в $H^{1}(X, \mathbb{Z})$, состоящему из стандартных образующих кольца Орлика-Соломона [6].

1) Понятие невырожденной структуры инцидентности по существу эквивалентно понятию простого матроида ранга 3 на множестве прямых. 
Группа $\langle\mathscr{R}\rangle$ порождена элементами $r(i, p)$ (являющимися коммутаторами), а также элементами вида $\left[w_{i_{1}}^{ \pm 1},\left[w_{i_{2}}^{ \pm 1}, \ldots,\left[w_{i_{k}}^{ \pm 1}, r(i, P)\right] \ldots\right]\right]$ (здесь квадратные скобки обозначают групповой коммутатор). Поэтому $\langle\mathscr{R}\rangle \subset \gamma_{2} F$. Легко видеть, что образы элементов из $\mathscr{R}$ в $L_{2}$ имеют вид $\bar{r}(i, p)=\left[x_{i}, \sum_{j: p \prec l_{j}} x_{j}\right]$ (здесь квадратные скобки обозначают лиевский коммутатор); таким образом, они зависят только от $C$.

В дальнейшем нам понадобится более широкий класс групп $G$. Пусть теперь $\mathscr{R}=\left\{r(i, p) \mid(i, p) \in \mathscr{A}, i \neq \min \left\{j \mid l_{j} \succ p\right\}\right\}$ - множество произвольных элементов из $\gamma_{2} F$, удовлетворяющих условию, что образы элементов $r(i, p)$ в $L_{2}$ - те же $\bar{r}(i, p)$, что и выше. Назовем такое множество $\mathscr{R}$ допустимым для $C$. Как и выше, полагаем $G=G(\mathscr{R})=F /\langle\mathscr{R}\rangle$. Поскольку $\langle\mathscr{R}\rangle \subset \gamma_{2} F$, каноническая проекция $F \rightarrow H$ пропускается через однозначно определенный сюръективный гомоморфизм $G \rightarrow H$. Ядро этого гомоморфизма, очевидно, совпадает с коммутантом группы $G$. Нас будут интересовать условия, при которых между двумя группами вида $G(\mathscr{R})$ (с различными допустимыми $\mathscr{R})$ существует изоморфизм, согласованный с определенными выше проекциями $G(\mathscr{R}) \rightarrow H$.

Нижний центральный ряд группы $F$ задает на подгруппе $\langle\mathscr{R}\rangle \subset F$ фильтрацию $\left(\langle\mathscr{R}\rangle \cap \gamma_{k} F\right)$, а на факторгруппе $G=F /\langle\mathscr{R}\rangle$ - фильтрацию, совпадающую с ее нижним центральным рядом $\left(\gamma_{k} G\right)$. Через $\operatorname{gr}_{k}\langle\mathscr{R}\rangle$ и $\operatorname{gr}_{k} G$ мы будем обозначать присоединенные градуированные факторы этих фильтраций. По определению допустимого множества $\mathscr{R}$ абелева группа $R_{2}=\operatorname{gr}_{2}\langle\mathscr{R}\rangle \subseteq L_{2}$ порождена элементами $\bar{r}(i, p)=\left[x_{i}, \sum_{j: p \prec l_{j}} x_{j}\right]$ и, таким образом, однозначно определяется структурой $C$. Докажем, что и группа $R_{3}=\operatorname{gr}_{3}\langle\mathscr{R}\rangle \subseteq L_{3}$ однозначно определяется структурой $C$.

Предложение 2.1. Для любого допустимого множества $\mathscr{R}$ имеет место равенство $R_{3}=\left[H, R_{2}\right]$ в алгебре Ли $L=\operatorname{gr} F$.

Доказательство. Прежде всего, заметим, что $\bar{r}(i, p)$ линейно независимы в $L_{2}$. Из элементов вида $\left[w_{j}, r(i, P)\right]$ выберем такие $u_{1}, \ldots, u_{m}$, чтобы их образы образовывали базис в $\left[H, R_{2}\right]$. Аналогично теореме $\Phi$. Холла о базисе (см. $[5$, теорема $5.13 \mathrm{~A}]$ ), легко доказать, что любой элемент группы $\langle\mathscr{R}\rangle$ однозначно представляется в виде $\prod_{r \in \mathscr{R}} r^{c_{r}} \cdot \prod_{i=1}^{m} u_{i}^{d_{i}} \cdot z$, где $c_{r}, d_{i} \in \mathbb{Z}, z \in\langle\mathscr{R}\rangle \cap \gamma_{4} F$ (порядок, в котором элементы $r \in \mathscr{R}$ встречаются в произведении $\prod_{r \in \mathscr{R}} r^{c_{r}}$,

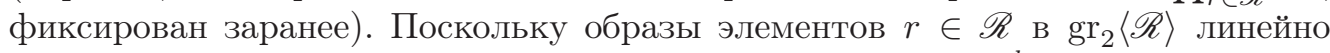
независимы, любой элемент из $\langle\mathscr{R}\rangle \cap \gamma_{3} F$ имеет вид $\prod_{i=1}^{m} u_{i}^{d_{i}} \cdot z$. Отсюда сразу следует, что $R_{3}=\left[H, R_{2}\right]$.

Положим $P_{2}=L_{2} / R_{2}$ и $P_{3}=L_{3} / R_{3}$. Если группа $G$ задана образующими и соотношениями как $G(\mathscr{R})$ с некоторым допустимым $\mathscr{R}$, то тем самым задан изоморфизм $G / \gamma_{2} G=\mathrm{gr}_{1} G \cong H$. Легко видеть, что коль скоро этот изоморфизм задан, изоморфизмы $\gamma_{2} G / \gamma_{3} G=\operatorname{gr}_{2} G \cong P_{2}$ и $\gamma_{3} G / \gamma_{4} G=\operatorname{gr}_{3} G \cong P_{3}$ однозначно определены. В этом смысле $\gamma_{2} G / \gamma_{3} G$ и $\gamma_{3} G / \gamma_{4} G$ канонически определяются структурой $C$.

Группа $G / \gamma_{3} G$ является расширением группы $H$ (свободной абелевой группы) с помощью тривиального $H$-модуля $P_{2}$. Такие расширения параметризуются группой $H^{2}\left(H, P_{2}\right) \cong \operatorname{Hom}\left(\Lambda^{2} H, P_{2}\right)$. Но поскольку $\Lambda^{2} H \cong L_{2}$, мы имеем естественную проекцию $\chi_{2}: \Lambda^{2} H \rightarrow P_{2}$. Легко видеть, что $\chi_{2}-$ это в точности элемент из $H^{2}\left(H, P_{2}\right)$, соответствующий групповому расширению 
$1 \rightarrow P_{2} \rightarrow G / \gamma_{3} G \rightarrow H \rightarrow 1$. Поэтому $G / \gamma_{3} G$ определяется структурой инцидентности канонически с точностью до автоморфизма, тривиального на $H$ и на $P_{2}$. Группа таких автоморфизмов изоморфна $\operatorname{Hom}\left(H, P_{2}\right)$.

Группа $M=\gamma_{2} G / \gamma_{4} G$ абелева, но структура $H$-модуля на ней нетривиальна. Рассуждения, подобные тем, что мы использовали для $G / \gamma_{3} G$, показывают, что этот модуль также определяется структурой $C$ канонически с точностью до действия абелевой группы $\operatorname{Hom}\left(P_{2}, P_{3}\right)$.

Рассмотрим групповое расширение $1 \rightarrow M \rightarrow G / \gamma_{4} G \rightarrow H \rightarrow 1$. Оно соответствует некоторому элементу $\chi_{3} \in H^{2}(H, M)$. Из короткой точной последовательности $H$-модулей $0 \rightarrow P_{3} \rightarrow M \rightarrow P_{2} \rightarrow 0$ мы получаем длинную точную последовательность

$$
\cdots \rightarrow H^{1}\left(H, P_{2}\right) \stackrel{\delta}{\rightarrow} H^{2}\left(H, P_{3}\right) \stackrel{\alpha}{\rightarrow} H^{2}(H, M) \stackrel{\beta}{\rightarrow} H^{2}\left(H, P_{2}\right) \rightarrow \ldots
$$

Перепишем ее в следующем виде:

$$
\cdots \rightarrow \operatorname{Hom}\left(H, P_{2}\right) \stackrel{\delta}{\rightarrow} \operatorname{Hom}\left(\Lambda^{2} H, P_{3}\right) \stackrel{\alpha}{\rightarrow} H^{2}(H, M) \stackrel{\beta}{\rightarrow} \operatorname{Hom}\left(\Lambda^{2} H, P_{2}\right) \rightarrow \ldots
$$

Мы имеем равенство $\beta\left(\chi_{3}\right)=\chi_{2}$, поэтому $\chi_{3}$ есть элемент множества $\beta^{-1}\left(\chi_{2}\right)$, которое является главным однородным пространством над абелевой группой $\operatorname{Hom}\left(\Lambda^{2} H, P_{3}\right) / \delta \operatorname{Hom}\left(H, P_{2}\right)$.

Напомним, что $H$-модуль $M$ определяется по $C$ лишь с точностью до действия абелевой группы $\operatorname{Hom}\left(P_{2}, P_{3}\right)$, поэтому только орбита $\bar{\chi}_{3}$ элемента $\chi_{3}$ относительно этого действия имеет инвариантный смысл. Множество орбит $Y=\beta^{-1}\left(\chi_{2}\right) / \operatorname{Hom}\left(P_{2}, P_{3}\right)$ является главным однородным пространством над группой $T=\operatorname{Hom}\left(R_{2}, P_{3}\right) / \bar{\delta} \operatorname{Hom}\left(H, P_{2}\right)$, где $\bar{\delta}-$ это композиция $\delta$ с естественной проекцией $\operatorname{Hom}\left(\Lambda^{2} H, P_{3}\right) \rightarrow \operatorname{Hom}\left(R_{2}, P_{3}\right)$. Мы видим, что $Y, T$ и действие $T$ на $Y$ определяются структурой $C$ канонически, так что любой выбор допустимого множества $\mathscr{R}$ задает корректно определенный элемент $\bar{\chi}_{3}(\mathscr{R}) \in Y$.

Предположим теперь, что заданы два допустимых множества $\mathscr{R}$ и $\mathscr{R}^{\prime}$. Рассмотрим соответствующие группы $G=G(\mathscr{R})$ и $G^{\prime}=G\left(\mathscr{R}^{\prime}\right)$. Пусть $\kappa\left(\mathscr{R}, \mathscr{R}^{\prime}\right)=$ $\bar{\chi}_{3}\left(\mathscr{R}^{\prime}\right)-\bar{\chi}_{3}(\mathscr{R}) \in T$. Из определений ясно, что $\kappa\left(\mathscr{R}, \mathscr{R}^{\prime \prime}\right)=\kappa\left(\mathscr{R}, \mathscr{R}^{\prime}\right)+\kappa\left(\mathscr{R}^{\prime}, \mathscr{R}^{\prime \prime}\right)$ для любых допустимых $\mathscr{R}, \mathscr{R}^{\prime}$ и $\mathscr{R}^{\prime \prime}$.

Обе группы $G / \gamma_{2} G$ и $G^{\prime} / \gamma_{2} G^{\prime}$ канонически изоморфны $H$. Поэтому мы имеем канонический изоморфизм $\lambda_{1}: G / \gamma_{2} G \rightarrow G^{\prime} / \gamma_{2} G^{\prime}$. Как отмечалось выше, изоморфизм $\lambda_{2}: G / \gamma_{3} G \rightarrow G^{\prime} / \gamma_{3} G^{\prime}$, продолжающий $\lambda_{1}$, существует всегда, хотя он и не определен канонически.

Теорема 2.2. Изоморфизм $\lambda_{3}: G / \gamma_{4} G \rightarrow G^{\prime} / \gamma_{4} G^{\prime}$, продолэсающий $\lambda_{1}$, существует тогда и только тогда, когда $к\left(\mathscr{R}, \mathscr{R}^{\prime}\right)=0$ в $T$.

Доказательство. Как мы показали, алгебры Ли $\operatorname{gr}\left(G / \gamma_{4} G\right)$ и $\operatorname{gr}\left(G^{\prime} / \gamma_{4} G^{\prime}\right)$ канонически изоморфны и порождаются $G / \gamma_{2} G$ и $G^{\prime} / \gamma_{2} G^{\prime}$ соответственно. Поэтому если $\lambda_{3}$ существует, то gr $\lambda_{3}$ определен однозначно и совпадает с каноническим изоморфизмом. Следовательно, $\kappa\left(\mathscr{R}, \mathscr{R}^{\prime}\right)=0$.

В другую сторону, если $\kappa\left(\mathscr{R}, \mathscr{R}^{\prime}\right)=0$, то расширения $1 \rightarrow M \rightarrow G / \gamma_{4} G \rightarrow$ $H \rightarrow 1$ и $1 \rightarrow M \rightarrow G^{\prime} / \gamma_{4} G^{\prime} \rightarrow H \rightarrow 1$ отличаются на некоторый автоморфизм $H$-модуля $M$; поэтому после применения этого автоморфизма они становятся эквивалентными. Следовательно, существует изоморфизм $\lambda_{3}$, продолжающий $\lambda_{1}$. 
Чтобы использовать инвариант, предоставляемый теоремой 2.2, мы должны еще описать $P_{2}$ и $P_{3}$. В общем случае можно сказать следующее.

Отождествим $L_{2}$ с $\Lambda^{2} H$. Обозначим через $\left(x_{i}^{*}\right)$ базис в $H^{*}$, двойственный к базису $\left(x_{i}\right)$ в $H$. Тогда $L_{2}^{*}=\Lambda^{2} H^{*}$ и $R_{2}^{\perp} \subset \Lambda^{2} H^{*}$ порождается следующими элементами: $\omega_{i j k}=\left(x_{i}^{*}-x_{j}^{*}\right) \wedge\left(x_{j}^{*}-x_{k}^{*}\right)=x_{i}^{*} \wedge x_{j}^{*}+x_{j}^{*} \wedge x_{k}^{*}+x_{k}^{*} \wedge x_{i}^{*}$ при $l_{i} \succ l_{j} \cap l_{k} \in \mathscr{P}_{0}$ и $\omega_{i j}=x_{i}^{*} \wedge x_{j}^{*}$ при $l_{0} \succ l_{i} \cap l_{j}$. Легко проверить, что $\left(R_{2}^{\perp}\right)^{\perp}=R_{2}$, поэтому $P_{2}$ является свободной абелевой группой и $P_{2}^{*} \cong R_{2}^{\perp}$. Следовательно, мы можем рассматривать определенные выше формы $\omega_{i j k}$ и $\omega_{i j}$ как элементы группы $P_{2}^{*}$.

Мы имеем точную последовательность абелевых групп

$$
0 \rightarrow \Lambda^{3} H \stackrel{d}{\rightarrow} H \otimes L_{2} \stackrel{c}{\rightarrow} L_{3} \rightarrow 0,
$$

где $d(x \wedge y \wedge z)=x \otimes[y, z]+y \otimes[z, x]+z \otimes[x, y]$ и $c(x \otimes f)=[x, f]$. Как мы знаем, $R_{3}=\left[H, R_{2}\right]$, поэтому $R_{3}=c\left(H \otimes R_{2}\right)$. Из двойственной точной последовательности

$$
0 \leftarrow \Lambda^{3} H^{*} \stackrel{d^{*}}{\longleftarrow} H^{*} \otimes \Lambda^{2} H^{*} \stackrel{c^{*}}{\leftarrow} L_{3}^{*} \leftarrow 0
$$

мы видим, что $R_{3}^{\perp}=\left.\operatorname{Ker} d^{*}\right|_{H^{*} \otimes R_{2}^{\perp}}$. Следовательно, $R_{3}^{\perp}$ содержит элементы вида $S_{i j k}=\left(x_{i}^{*}-x_{j}^{*}\right) \otimes \omega_{i j k}$ при $l_{i} \succ l_{j} \cap l_{k} \in \mathscr{P}_{0}$ и $S_{i j}=x_{i}^{*} \otimes \omega_{i j}$ при $l_{0} \succ$ $l_{i} \cap l_{j}$. Я не утверждаю, что эти элементы порождают $R_{3}^{\perp}-$ как мы увидим в следующем параграфе, это не обязательно так, - но я не знаю никакого общего описания остальных элементов из $R_{3}^{\perp}$.

Если расшифровать данное выше в гомологических терминах определение $\kappa\left(\mathscr{R}, \mathscr{R}^{\prime}\right)$, получается следующий явный алгоритм для его вычисления.

Пусть $\mathscr{R}=\left\{r(i, p) \mid(i, p) \in \mathscr{A}, i \neq \min \left\{j \mid l_{j} \succ p\right\}\right\}$ и $\mathscr{R}^{\prime}=\left\{r^{\prime}(i, p) \mid(i, p) \in\right.$ $\left.\mathscr{A}, i \neq \min \left\{j \mid l_{j} \succ p\right\}\right\}$ - произвольные допустимые множества. Множество $\overline{\mathscr{R}}=\left\{\bar{r}(i, p) \mid(i, p) \in \mathscr{A}, i \neq \min \left\{j \mid l_{j} \succ p\right\}\right\}$ образует базис в $R_{2}$. Поставим в соответствие каждому элементу $\bar{r}(i, p) \in \overline{\mathscr{R}}$ элемент $t(i, p)=\left(r^{\prime}(i, p)\right)^{-1} r(i, p) \in F$. Поскольку $r(i, p), r^{\prime}(i, p) \in \gamma_{2} F$, причем образы их в $\operatorname{gr}_{2} F$ совпадают (и равны $\bar{r}(i, p))$, то $t(i, p) \in \gamma_{3} F$. Рассмотрим гомоморфизм $R_{2} \rightarrow P_{3}$, определенный на базисе $\overline{\mathscr{R}}$ так: каждый элемент $\bar{r}(i, p)$ переходит в образ элемента $t(i, p)$ в $P_{3}=\left(\mathrm{gr}_{3} F\right) / R_{3}$. Образ этого гомоморфизма в $T=\operatorname{Hom}\left(R_{2}, P_{3}\right) / \bar{\delta} \operatorname{Hom}\left(H, P_{2}\right)-$ это и есть $\kappa\left(\mathscr{R}, \mathscr{R}^{\prime}\right)$.

Далее нам также понадобится явная формула для отображения $\bar{\delta}$ :

$$
\bar{\delta} f(\bar{r}(i, p))=\left[f\left(x_{i}\right), \sum_{j: p \prec l_{j}} x_{j}\right]+\left[x_{i}, \sum_{j: p \prec l_{j}} f\left(x_{j}\right)\right]
$$

для любого $f \in \operatorname{Hom}\left(H, P_{2}\right)$.

Посмотрим, как можно описать $\kappa\left(\mathscr{R}, \mathscr{R}^{\prime}\right)$ в случае, когда $\mathscr{R}=\mathscr{R}(g)$ и $\mathscr{R}^{\prime}=$ $\mathscr{R}\left(g^{\prime}\right)$ для некоторых отображений $g, g^{\prime}: \mathscr{A} \rightarrow F$.

Предложение 2.3. Если $g(i, p) \equiv g^{\prime}(i, p)\left(\bmod \gamma_{2} F\right)$ для любых $i=1, \ldots, n$ и $p \prec l_{i}$, то $\kappa\left(\mathscr{R}, \mathscr{R}^{\prime}\right)=0$. Иными словами, $\bar{\chi}_{3}(\mathscr{R}(g))$ зависит только от $\bar{g}: \mathscr{A} \rightarrow H$.

Доказательство. Действительно, в этом случае $w_{i}(p) \equiv w_{i}^{\prime}(p)\left(\bmod \gamma_{3} F\right)$ и, следовательно, $r(i, p) \equiv r^{\prime}(i, p)\left(\bmod \gamma_{4} F\right)$. Поэтому $(r(i, p))^{-1} r^{\prime}(i, p) \in \gamma_{4} F$ и $\kappa\left(\mathscr{R}, \mathscr{R}^{\prime}\right)=0$. 
Рассмотрим абелеву группу $A=H^{\mathscr{A}}$. Для $a \in A$ определим $\tilde{\tau} a \in \operatorname{Hom}\left(R_{2}, P_{3}\right)$, полагая

$$
\tilde{\tau} a(\bar{r}(i, p))=\left[\left[x_{i}, a(i, p)\right], \sum_{j: p \prec l_{j}} x_{j}\right]+\left[x_{i}, \sum_{j: p \prec l_{j}}\left[x_{j}, a(j, p)\right]\right]+R_{3} .
$$

Пусть $\tau a=\tilde{\tau} a+\bar{\delta} \operatorname{Hom}\left(H, P_{2}\right)$.

Предложение 2.4. Если $\mathscr{R}=\mathscr{R}(g) u \mathscr{R}^{\prime}=\mathscr{R}\left(g^{\prime}\right)$, mo $\kappa\left(\mathscr{R}, \mathscr{R}^{\prime}\right)=\tau\left(\bar{g}-\bar{g}^{\prime}\right)$.

Доказательство следует из приведенного выше алгоритма для вычисления $\kappa\left(\mathscr{R}, \mathscr{R}^{\prime}\right)$.

Было бы интересно найти $\operatorname{Ker} \tau$. Вот несколько частичных результатов в этом направлении.

Определим следующие элементы из $A: a_{i, p}^{(0)}$ при $i \in\{1, \ldots, n\}, p \in \mathscr{P}_{0}, p \prec l_{i}$; $a_{i, p}^{(1)}$ при $i \in\{1, \ldots, n\}, p \in \mathscr{P}_{0} ; a_{i, p_{1}, p_{2}}^{(2)}$ для $i \in\{1, \ldots, n\}, p_{1}, p_{2} \in \mathscr{P}_{0}, p_{1}, p_{2} \prec l_{i}$ $\left(p_{1}\right.$ и $p_{2}$ не обязательно различны), с помощью формул

$$
a_{i, p}^{(0)}(j, q)=\delta_{i, j} \delta_{p, q} x_{i}, \quad a_{i, p}^{(1)}(j, q)=\delta_{p, q} x_{i}, \quad a_{i, p_{1}, p_{2}}^{(2)}(j, q)=\delta_{i, j} \delta_{p_{1}, q} \sum_{k: p_{2} \succ l_{k}} x_{k} .
$$

Обозначим через $U$ порожденную ими подгруппу в $A$.

Предложение 2.5. $U \subseteq \operatorname{Ker} \tilde{\tau}$.

Доказательство. Утверждение проверяется прямым вычислением.

Пусть $B$ - подгруппа в $A$, состоящая из функций, не зависящих от $p$.

Предложение 2.6. $B \subseteq \tilde{\tau}^{-1}\left(\bar{\delta} \operatorname{Hom}\left(H, P_{2}\right)\right)$.

Доказательство. Пусть $a \in B, a(i, p)=\sum_{j=1}^{n} k_{i j} x_{j}$. Определим элемент $f \in \operatorname{Hom}\left(H, P_{2}\right)$, полагая $f\left(x_{i}\right)=\sum_{j=1}^{n} k_{i j}\left[x_{i}, x_{j}\right]$. Тогда $\tilde{\tau} a=\bar{\delta}(f)$.

Положим $W=A /(U+B)$. Гомоморфизм $\tau$ является композицией проекции $\pi: A \rightarrow W$ и некоторого гомоморфизма $\bar{\tau}: W \rightarrow T$. Я не знаю, всегда ли гомоморфизм $\bar{\tau}$ инъективен, но в примере, который будет разобран в следующем параграфе, это так.

\section{§3. Вычисления для структуры Маклейна}

Матроид Маклейна $M L_{8}$ может быть определен как аффинная плоскость над $\mathbb{F}_{3}$, из которой выброшен один элемент. Прямые соответствующей структуры инцидентности $C_{8}$ занумерованы элементами из $\mathbb{F}_{3}^{2} \backslash\{(0,0)\}$, причем три прямые в $C_{8}$ пересекаются в одной точке тогда и только тогда, когда соответствующие элементы лежат на одной аффинной прямой (в смысле $\mathbb{F}_{3}^{2}$ ). Из этого описания видно, что группа автоморфизмов структуры $C_{8}$ изоморфна $G L_{2}\left(\mathbb{F}_{3}\right)$.

Занумеруем прямые структуры $C_{8}$ так, чтобы они соответствовали следующим точкам из $\mathbb{F}_{3}^{2}: l_{0} \leftrightarrow(2,2), l_{1} \leftrightarrow(0,1), l_{2} \leftrightarrow(1,0), l_{3} \leftrightarrow(2,0), l_{4} \leftrightarrow(2,1)$, $l_{5} \leftrightarrow(1,2), l_{6} \leftrightarrow(0,2), l_{7} \leftrightarrow(1,1)$. Тогда точки структуры $C_{8}$ имеют вид $p_{012}$, $p_{034}, p_{056}, p_{07}, p_{135}, p_{147}, p_{16}, p_{23}, p_{246}, p_{257}, p_{367}, p_{45}$, где $p_{i j k} \prec l_{i}, l_{j}, l_{k}$ и $p_{i j} \prec l_{i}, l_{j}$. Таким образом, $\mathscr{P}_{0}$ состоит из последних 8 из перечисленных выше точек. 
Легко проверить, что любая комплексная реализация структуры $C_{8}$ проективно эквивалентна реализации $\phi$ следующего вида:

$$
\begin{aligned}
& \phi l_{0}=\left\{\left(z_{0}: z_{1}: z_{2}\right) \in \mathbb{C} P^{2} \mid z_{0}=0\right\}, \\
& \phi l_{1}=\left\{\left(z_{0}: z_{1}: z_{2}\right) \in \mathbb{C} P^{2} \mid z_{1}=0\right\}, \\
& \phi l_{2}=\left\{\left(z_{0}: z_{1}: z_{2}\right) \in \mathbb{C} P^{2} \mid z_{1}=z_{0}\right\}, \\
& \phi l_{3}=\left\{\left(z_{0}: z_{1}: z_{2}\right) \in \mathbb{C} P^{2} \mid z_{2}=0\right\}, \\
& \phi l_{4}=\left\{\left(z_{0}: z_{1}: z_{2}\right) \in \mathbb{C} P^{2} \mid z_{2}=z_{0}\right\}, \\
& \phi l_{5}=\left\{\left(z_{0}: z_{1}: z_{2}\right) \in \mathbb{C} P^{2} \mid z_{2}+\omega z_{1}=0\right\}, \\
& \phi l_{6}=\left\{\left(z_{0}: z_{1}: z_{2}\right) \in \mathbb{C} P^{2} \mid z_{2}+\omega z_{1}=(\omega+1) z_{0}\right\}, \\
& \phi l_{7}=\left\{\left(z_{0}: z_{1}: z_{2}\right) \in \mathbb{C} P^{2} \mid(\omega+1) z_{1}+z_{2}=z_{0}\right\},
\end{aligned}
$$

где $\omega-$ некоторый корень многочлена $x^{2}+x+1$. Точки $\phi p$ для $p \in \mathscr{P}$ определяются как точки пересечения соответствующих прямых.

Обозначим через $\phi^{+}$(соответственно через $\phi^{-}$) реализацию структуры $C_{8}$, отвечающую корню $\omega=\exp (2 \pi i / 3)$ (соответственно корню $\omega=\exp (-2 \pi i / 3)$ ). Положим $G^{+}=\pi_{1}\left(X^{+}\right) / \gamma_{4} \pi_{1}\left(X^{+}\right)$и $G^{-}=\pi_{1}\left(X^{-}\right) / \gamma_{4} \pi_{1}\left(X^{-}\right)$.

Теорема 3.1. Не существует изоморфизма $G^{+} \rightarrow G^{-}$, такого, что индуцированный изоморфизм $H_{1}\left(X^{+}, \mathbb{Z}\right) \rightarrow H_{1}\left(X^{-}, \mathbb{Z}\right)$ переводит элементы канонического базиса группы $H_{1}\left(X^{+}, \mathbb{Z}\right)$ в соответствующие элементы канонического базиса группь $H_{1}\left(X^{-}, \mathbb{Z}\right)$.

Доказательство. Пусть $X^{ \pm}=\mathbb{C} P^{2} \backslash \bigcup \phi^{ \pm} l$. Применение алгоритма Арволы дает нам следующие наборы определяющих соотношений для $\pi_{1}\left(X^{ \pm}\right)$(в обоих случаях в свободной группе $F$ с образующими $\left.w_{1}, \ldots, w_{7}\right)$ :

$$
\begin{aligned}
& {\left[w_{5}, w_{3}, w_{1}\right], \quad\left[w_{7}, w_{4}, w_{1}\right], \quad\left[w_{6}, w_{1}\right], \quad\left[w_{3}, w_{2}\right], \quad\left[w_{6}, w_{4}, w_{2}\right],} \\
& {\left[w_{7}, w_{5}, w_{5}^{-1} w_{2} w_{5}\right], \quad\left[w_{7}, w_{6}, w_{6}^{-1} w_{3} w_{6}\right], \quad\left[w_{5}, w_{3} w_{6} w_{4} w_{6}^{-1} w_{3}^{-1}\right]}
\end{aligned}
$$

для $\pi_{1}\left(X^{+}\right)$и

$$
\begin{gathered}
{\left[w_{5}, w_{3}, w_{1}\right], \quad\left[w_{7}, w_{4}, w_{1}\right], \quad\left[w_{6}, w_{1}\right], \quad\left[w_{3}, w_{5}^{-1} w_{2} w_{5}\right], \quad\left[w_{7}^{-1} w_{6} w_{7}, w_{4}, w_{2}\right],} \\
{\left[w_{7}, w_{5}, w_{5}^{-1} w_{2} w_{5}\right], \quad\left[w_{7}, w_{6}, w_{6}^{-1} w_{3} w_{6}\right], \quad\left[w_{5}, w_{7} w_{4} w_{7}^{-1}\right]}
\end{gathered}
$$

для $\pi_{1}\left(X^{-}\right)$. Эти наборы соотношений отвечают отображениям $g^{ \pm}: \mathscr{A} \rightarrow F$, все значения которых равны 1 , кроме следующих:

$$
\begin{gathered}
g^{+}\left(3, p_{367}\right)=w_{6}, \quad g^{+}\left(4, p_{45}\right)=w_{6}^{-1} w_{3}^{-1}, \quad g^{+}\left(2, p_{257}\right)=w_{5}, \quad g^{-}\left(3, p_{367}\right)=w_{6}, \\
g^{-}\left(4, p_{45}\right)=w_{7}^{-1}, \quad g^{-}\left(2, p_{23}\right)=w_{5}, \quad g^{-}\left(2, p_{257}\right)=w_{5}, \quad g^{-}\left(6, p_{246}\right)=w_{7} .
\end{gathered}
$$

Положим $a_{0}=\bar{g}^{+}-\bar{g}^{-}: \mathscr{A} \rightarrow H$. Это отображение имеет лишь следующие ненулевые значения:

$$
a_{0}\left(4, p_{45}\right)=x_{7}-x_{6}-x_{3}, \quad a_{0}\left(2, p_{23}\right)=-x_{5}, \quad a_{0}\left(6, p_{246}\right)=-x_{7} .
$$

По теореме 2.2 и предложению 2.4 достаточно доказать, что $\tau a_{0} \neq 0$ в $T$.

Лемма 3.2. Образ отображения $a_{0}$ в $W$ ненулевой. 
Доказательство. Вначале мы вычислим $\widetilde{W}=A / U$. Так как любая образующая $a_{i, p}^{(\alpha)}$ группы $U$ обращается в нуль на $(j, q)$ с $q \neq p$, мы можем рассмотреть разложения $A=\bigoplus_{p \in \mathscr{P}_{0}} A_{p}, U=\bigoplus_{p \in \mathscr{P}_{0}} U_{p}$ и $\widetilde{W}=\bigoplus_{p \in \mathscr{P}_{0}} \widetilde{W}_{p}$, где $A_{p}, U_{p}$ и $\widetilde{W}_{p}$ определены очевидным образом. Для каждого $p$ вычисление легко проделать. Все $\widetilde{W}_{p}$ оказываются свободными абелевыми группами, поэтому $\widetilde{W}$ - тоже свободная абелева группа, и, таким образом, ее двойственная $\widetilde{W}^{*}$ естественно изоморфна $U^{\perp} \subset A^{*}$.

Пусть $e_{i j}(p) \in A^{*}$ определяются равенствами $\left(e_{i j}(p), a\right)=\left(x_{j}^{*}, a(i, p)\right)$ для $a \in A$, где $\left(x_{i}^{*}\right)$ - базис в $H^{*}$, двойственный каноническому базису $\left(x_{i}\right)$ в $H$. Базис в $U^{\perp}$ состоит из следующих элементов:

$$
\begin{gathered}
I\left(p_{135}\right)=e_{13}\left(p_{135}\right)-e_{15}\left(p_{135}\right)+e_{35}\left(p_{135}\right)-e_{31}\left(p_{135}\right)+e_{51}\left(p_{135}\right)-e_{53}\left(p_{135}\right), \\
I\left(p_{147}\right)=e_{14}\left(p_{147}\right)-e_{17}\left(p_{147}\right)+e_{47}\left(p_{147}\right)-e_{41}\left(p_{147}\right)+e_{71}\left(p_{147}\right)-e_{74}\left(p_{147}\right), \\
I\left(p_{367}\right)=e_{36}\left(p_{367}\right)-e_{37}\left(p_{367}\right)+e_{67}\left(p_{367}\right)-e_{63}\left(p_{367}\right)+e_{73}\left(p_{367}\right)-e_{76}\left(p_{367}\right), \\
I\left(p_{257}\right)=e_{25}\left(p_{257}\right)-e_{27}\left(p_{257}\right)+e_{57}\left(p_{257}\right)-e_{52}\left(p_{257}\right)+e_{72}\left(p_{257}\right)-e_{75}\left(p_{257}\right), \\
I\left(p_{246}\right)=e_{24}\left(p_{246}\right)-e_{26}\left(p_{246}\right)+e_{46}\left(p_{246}\right)-e_{42}\left(p_{246}\right)+e_{62}\left(p_{246}\right)-e_{64}\left(p_{246}\right), \\
J\left(p_{16}\right)=e_{12}\left(p_{16}\right)-e_{62}\left(p_{16}\right)+e_{64}\left(p_{16}\right)-e_{14}\left(p_{16}\right)+e_{17}\left(p_{16}\right)-e_{67}\left(p_{16}\right) \\
\quad+e_{63}\left(p_{16}\right)-e_{13}\left(p_{16}\right)+e_{15}\left(p_{16}\right)-e_{65}\left(p_{16}\right), \\
\quad+e_{52}\left(p_{45}\right)-e_{42}\left(p_{45}\right)+e_{46}\left(p_{45}\right)-e_{56}\left(p_{45}\right), \\
J\left(p_{45}\right)=e_{43}\left(p_{45}\right)-e_{53}\left(p_{45}\right)+e_{51}\left(p_{45}\right)-e_{41}\left(p_{45}\right)+e_{47}\left(p_{45}\right)-e_{57}\left(p_{45}\right) \\
\quad+e_{36}\left(p_{23}\right)-e_{26}\left(p_{23}\right)+e_{24}\left(p_{23}\right)-e_{34}\left(p_{23}\right), \\
J\left(p_{23}\right)=e_{21}\left(p_{23}\right)-e_{31}\left(p_{23}\right)+e_{35}\left(p_{23}\right)-e_{25}\left(p_{23}\right)+e_{27}\left(p_{23}\right)-e_{37}\left(p_{23}\right) \\
\left.\quad+e_{136}\right)
\end{gathered}
$$

$K_{1}\left(p_{135}\right)=e_{12}\left(p_{135}\right)+e_{36}\left(p_{135}\right)-e_{37}\left(p_{135}\right)+e_{57}\left(p_{135}\right)-e_{52}\left(p_{135}\right)-e_{56}\left(p_{135}\right)$, $K_{2}\left(p_{135}\right)=e_{14}\left(p_{135}\right)-e_{17}\left(p_{135}\right)+e_{37}\left(p_{135}\right)-e_{34}\left(p_{135}\right)-e_{36}\left(p_{135}\right)+e_{56}\left(p_{135}\right)$, $K_{1}\left(p_{147}\right)=e_{12}\left(p_{147}\right)+e_{13}\left(p_{147}\right)-e_{15}\left(p_{147}\right)-e_{43}\left(p_{147}\right)+e_{75}\left(p_{147}\right)-e_{72}\left(p_{147}\right)$, $K_{2}\left(p_{147}\right)=e_{12}\left(p_{147}\right)+e_{46}\left(p_{147}\right)-e_{42}\left(p_{147}\right)-e_{43}\left(p_{147}\right)+e_{73}\left(p_{147}\right)-e_{76}\left(p_{147}\right)$, $K_{1}\left(p_{367}\right)=e_{31}\left(p_{367}\right)-e_{34}\left(p_{367}\right)-e_{35}\left(p_{367}\right)+e_{65}\left(p_{367}\right)+e_{74}\left(p_{367}\right)-e_{71}\left(p_{367}\right)$, $K_{2}\left(p_{367}\right)=e_{34}\left(p_{367}\right)+e_{62}\left(p_{367}\right)-e_{64}\left(p_{367}\right)-e_{65}\left(p_{367}\right)+e_{75}\left(p_{367}\right)-e_{72}\left(p_{367}\right)$, $K_{1}\left(p_{257}\right)=e_{21}\left(p_{257}\right)+e_{53}\left(p_{257}\right)-e_{51}\left(p_{257}\right)-e_{56}\left(p_{257}\right)+e_{76}\left(p_{257}\right)-e_{73}\left(p_{257}\right)$, $K_{2}\left(p_{257}\right)=e_{24}\left(p_{257}\right)-e_{21}\left(p_{257}\right)-e_{26}\left(p_{257}\right)+e_{56}\left(p_{257}\right)+e_{71}\left(p_{257}\right)-e_{74}\left(p_{257}\right)$, $K_{1}\left(p_{246}\right)=e_{21}\left(p_{246}\right)+e_{47}\left(p_{246}\right)-e_{41}\left(p_{246}\right)-e_{43}\left(p_{246}\right)+e_{63}\left(p_{246}\right)-e_{67}\left(p_{246}\right)$, $K_{2}\left(p_{246}\right)=e_{25}\left(p_{246}\right)-e_{27}\left(p_{246}\right)+e_{43}\left(p_{246}\right)+e_{67}\left(p_{246}\right)-e_{63}\left(p_{246}\right)-e_{65}\left(p_{246}\right)$.

Рассмотрим следующую линейную функцию $t: \widetilde{W} \rightarrow \mathbb{Z} / 3 \mathbb{Z}$ :

$$
t=2 I\left(p_{367}\right)+J\left(p_{45}\right)+K_{1}\left(p_{135}\right)-K_{2}\left(p_{147}\right)+K_{1}\left(p_{257}\right)-K_{1}\left(p_{246}\right)+3 \mathbb{Z} .
$$

Несложно проверить, что $\left.t\right|_{B}=0$ и $t\left(a_{0}\right)=1$. Поэтому образ элемента $a_{0}$ в $W=\widetilde{W} / B$ не равен нулю.

Лемма 3.3. $U=\operatorname{Ker} \tilde{\tau}$ для структуры $C_{8}$. 
Доказательство. Чтобы доказать это, изучим сначала $P_{3}$. Вычисления показывают, что базис в $R_{3}^{\perp}$ состоит из элементов $S_{i j}=x_{i}^{*} \otimes x_{i}^{*} \wedge x_{j}^{*}$ для $l_{0} \succ l_{i} \cap l_{j}$, где $i<j, \quad S_{i j k}=\left(x_{i}^{*}-x_{j}^{*}\right) \otimes\left(x_{i}^{*}-x_{j}^{*}\right) \wedge\left(x_{j}^{*}-x_{k}^{*}\right)$ для $l_{i} \succ l_{j} \cap l_{k} \in \mathscr{P}_{0}$, где $i<j$, $i<k$, и следующих добавочных элементов:

$$
\begin{aligned}
& T_{0}=x_{7}^{*} \otimes\left(x_{1}^{*}-x_{3}^{*}\right) \wedge\left(x_{3}^{*}-x_{5}^{*}\right)+\left(x_{2}^{*}-x_{3}^{*}\right) \otimes\left(x_{1}^{*}-x_{4}^{*}\right) \wedge\left(x_{4}^{*}-x_{7}^{*}\right) \\
&+\left(x_{4}^{*}-x_{5}^{*}\right) \otimes\left(x_{3}^{*}-x_{6}^{*}\right) \wedge\left(x_{6}^{*}-x_{7}^{*}\right)+\left(x_{1}^{*}-x_{6}^{*}\right) \otimes\left(x_{2}^{*}-x_{5}^{*}\right) \wedge\left(x_{5}^{*}-x_{7}^{*}\right) \\
&-x_{7}^{*} \otimes\left(x_{2}^{*}-x_{4}^{*}\right) \wedge\left(x_{4}^{*}-x_{6}^{*}\right)+\left(x_{4}^{*}-x_{5}^{*}\right) \otimes x_{1}^{*} \wedge x_{2}^{*} \\
&-\left(x_{1}^{*}-x_{6}^{*}\right) \otimes x_{3}^{*} \wedge x_{4}^{*}+\left(x_{2}^{*}-x_{3}^{*}\right) \otimes x_{5}^{*} \wedge x_{6}^{*}, \\
& T_{1}=x_{7}^{*} \otimes\left(x_{1}^{*}-x_{3}^{*}\right) \wedge\left(x_{3}^{*}-x_{5}^{*}\right)-x_{3}^{*} \otimes\left(x_{1}^{*}-x_{4}^{*}\right) \wedge\left(x_{4}^{*}-x_{7}^{*}\right) \\
&-x_{5}^{*} \otimes\left(x_{3}^{*}-x_{6}^{*}\right) \wedge\left(x_{6}^{*}-x_{7}^{*}\right)+x_{1}^{*} \otimes\left(x_{2}^{*}-x_{5}^{*}\right) \wedge\left(x_{5}^{*}-x_{7}^{*}\right) \\
&-\left(x_{5}^{*}-x_{7}^{*}\right) \otimes x_{1}^{*} \wedge x_{2}^{*}-\left(x_{1}^{*}-x_{7}^{*}\right) \otimes x_{3}^{*} \wedge x_{4}^{*}-\left(x_{3}^{*}-x_{7}^{*}\right) \otimes x_{5}^{*} \wedge x_{6}^{*}, \\
& T_{2}=x_{2}^{*} \otimes\left(x_{1}^{*}-x_{3}^{*}\right) \wedge\left(x_{3}^{*}-x_{5}^{*}\right)+\left(x_{2}^{*}-x_{5}^{*}\right) \otimes\left(x_{3}^{*}-x_{6}^{*}\right) \wedge\left(x_{6}^{*}-x_{7}^{*}\right) \\
& \quad+\left(x_{3}^{*}-x_{6}^{*}\right) \otimes\left(x_{2}^{*}-x_{5}^{*}\right) \wedge\left(x_{5}^{*}-x_{7}^{*}\right)-x_{3}^{*} \otimes\left(x_{2}^{*}-x_{4}^{*}\right) \wedge\left(x_{4}^{*}-x_{6}^{*}\right) \\
& \quad+\left(x_{3}^{*}-x_{5}^{*}\right) \otimes x_{1}^{*} \wedge x_{2}^{*}-\left(x_{2}^{*}-x_{6}^{*}\right) \otimes x_{3}^{*} \wedge x_{4}^{*}+\left(x_{2}^{*}-x_{3}^{*}\right) \otimes x_{5}^{*} \wedge x_{6}^{*}, \\
& T_{3}=x_{6}^{*} \otimes\left(x_{1}^{*}-x_{3}^{*}\right) \wedge\left(x_{3}^{*}-x_{5}^{*}\right)-\left(x_{3}^{*}-x_{6}^{*}\right) \otimes\left(x_{1}^{*}-x_{4}^{*}\right) \wedge\left(x_{4}^{*}-x_{7}^{*}\right) \\
& \quad-\left(x_{1}^{*}-x_{4}^{*}\right) \otimes\left(x_{3}^{*}-x_{6}^{*}\right) \wedge\left(x_{6}^{*}-x_{7}^{*}\right)-x_{1}^{*} \otimes\left(x_{2}^{*}-x_{4}^{*}\right) \wedge\left(x_{4}^{*}-x_{6}^{*}\right) \\
& \quad+\left(x_{4}^{*}-x_{6}^{*}\right) \otimes x_{1}^{*} \wedge x_{2}^{*}-\left(x_{1}^{*}-x_{6}^{*}\right) \otimes x_{3}^{*} \wedge x_{4}^{*}+\left(x_{1}^{*}-x_{3}^{*}\right) \otimes x_{5}^{*} \wedge x_{6}^{*}, \\
& T_{4}=x_{4}^{*} \otimes\left(x_{1}^{*}-x_{3}^{*}\right) \wedge\left(x_{3}^{*}-x_{5}^{*}\right)+\left(x_{2}^{*}-x_{5}^{*}\right) \otimes\left(x_{1}^{*}-x_{4}^{*}\right) \wedge\left(x_{4}^{*}-x_{7}^{*}\right) \\
& \quad+\left(x_{1}^{*}-x_{4}^{*}\right) \otimes\left(x_{2}^{*}-x_{5}^{*}\right) \wedge\left(x_{5}^{*}-x_{7}^{*}\right)-x_{5}^{*} \otimes\left(x_{2}^{*}-x_{4}^{*}\right) \wedge\left(x_{4}^{*}-x_{6}^{*}\right) \\
& \quad+\left(x_{4}^{*}-x_{5}^{*}\right) \otimes x_{1}^{*} \wedge x_{2}^{*}-\left(x_{1}^{*}-x_{5}^{*}\right) \otimes x_{3}^{*} \wedge x_{4}^{*}+\left(x_{2}^{*}-x_{4}^{*}\right) \otimes x_{5}^{*} \wedge x_{6}^{*} .
\end{aligned}
$$

Более того, $\left(R_{3}^{\perp}\right)^{\perp}=R_{3}$, так что $P_{3}$ является свободной абелевой группой и $P_{3}^{*} \cong R_{3}^{\perp}$. Поэтому достаточно показать, что $U^{\perp}=\operatorname{Im} \tilde{\tau}^{*}$.

Отождествим $\left(\operatorname{Hom}\left(R_{2}, P_{3}\right)\right)^{*}$ с $R_{2} \otimes P_{3}^{*}$. Легко видеть, что

$$
\begin{gathered}
I\left(p_{135}\right)=\tilde{\tau}^{*}\left(r\left(1, p_{135}\right) \otimes S_{135}\right), \quad I\left(p_{147}\right)=\tilde{\tau}^{*}\left(r\left(1, p_{147}\right) \otimes S_{147}\right), \\
J\left(p_{16}\right)=\tilde{\tau}^{*}\left(r\left(1, p_{16}\right) \otimes T_{3}\right), \quad K_{1}\left(p_{135}\right)=\tilde{\tau}^{*}\left(r\left(1, p_{135}\right) \otimes\left(T_{0}-T_{3}\right)\right), \\
K_{2}\left(p_{135}\right)=\tilde{\tau}^{*}\left(r\left(1, p_{135}\right) \otimes T_{3}\right), \quad K_{1}\left(p_{147}\right)=-\tilde{\tau}^{*}\left(r\left(1, p_{147}\right) \otimes T_{0}\right), \\
K_{2}\left(p_{147}\right)=-\tilde{\tau}^{*}\left(r\left(1, p_{147}\right) \otimes T_{3}\right) .
\end{gathered}
$$

Поэтому $\left(U^{\perp}\right)_{p} \subset \operatorname{Im} \tilde{\tau}^{*}$ для $p=p_{135}, p_{147}, p_{16}$. Теперь мы можем воспользоваться тем, что группа, порожденная перестановками $(16)(25)(34)$ и $(135)(246)$, действует на $C_{8}$ автоморфизмами, чтобы доказать то же самое для любого $p \in \mathscr{P}_{0}$. Отсюда следует, что $U^{\perp} \subset \operatorname{Im} \tilde{\tau}^{*}$. Комбинируя это с предложением 2.5, мы получаем утверждение доказываемой леммы.

Лемма 3.4. $B=\tilde{\tau}^{-1}\left(\bar{\delta} \operatorname{Hom}\left(H, P_{2}\right)\right)$ для структуръ $C_{8}$.

Доказательство. Покажем сначала, что если $\bar{\delta} f \in \tilde{\tau} A$ для некоторого $f \in$ $\operatorname{Hom}\left(H, P_{2}\right)$, то

(1) $\left(\omega_{i j}, f\left(x_{k}\right)\right)=0$ для любых $i, j, k$, таких, что $l_{i} \cap l_{j} \prec l_{0}, k \neq i, j$, и

(2) $\left(\omega_{i j k}, f\left(x_{m}\right)\right)=0$ для любых $i, j, k, m$, таких, что $l_{i} \succ l_{j} \cap l_{k} \in \mathscr{P}_{0}$, $m \neq i, j, k$. 
Предположим, что утверждение (1) не выполнено; тогда, поскольку никакие четыре различные прямые из $C_{8}$ не пересекаются в одной точке, мы имеем $p=l_{i} \cap l_{k} \in \mathscr{P}_{0}$ и $\left(S_{i j}, \bar{\delta} f(r(k, p))\right)=\left(\omega_{i j}, f\left(x_{k}\right)\right) \neq 0$. Но $\left(S_{i j}, \tilde{\tau} a(r(k, p))\right)=0$ для любых $a \in A$; поэтому утверждение (1) должно выполняться.

Предположим теперь, что утверждение (2) не выполнено; тогда либо $p=$ $l_{i} \cap l_{m} \in \mathscr{P}_{0}$, либо $p^{\prime}=l_{j} \cap l_{m} \in \mathscr{P}_{0}$. Используя симметрию относительно порядка $(i j k)$, мы можем считать, что $p=l_{i} \cap l_{k} \in \mathscr{P}_{0}$. Тогда $\left(S_{i j k}, \bar{\delta} f(r(m, p))\right)=$ $\left(\omega_{i j k}, f\left(x_{m}\right)\right) \neq 0$. Но $\left(S_{i j k}, \tilde{\tau} a(r(m, p))\right)=0$ для любых $a \in A$; поэтому утверждение (2) тоже должно выполняться.

Следовательно, мы можем выбрать $\tilde{f} \in \operatorname{Hom}\left(H, \Lambda^{2} H\right)$ с $f(x)=\tilde{f}(x)+R_{2}$ так, чтобы $\tilde{f}\left(x_{k}\right)=x_{k} \wedge b(k)$ для некоторого $b:\{1, \ldots, 7\} \rightarrow H$. Отсюда вытекает, что $\bar{\delta} f=\tilde{\tau} a$, где $a \in B, a(k, p)=b(k)$ для любых $k$ и $p$.

Из последних двух лемм следует, что $\bar{\tau}: W \rightarrow T$ является вложением, поэтому $\tau a_{0} \neq 0$. Теорема 3.1 доказана.

Заметим, что в процессе вычислений мы предъявили ненулевой гомоморфный образ различающего инварианта $\tau a_{0}$ в группе $\mathbb{Z} / 3 \mathbb{Z}$. Небольшие дополнительные вычисления показывают, что и сам элемент $\tau a_{0}$ имеет порядок 3 в $T$ и, тем самым, принадлежит подгруппе кручения. Это не случайно - при тензорном умножении на $\mathbb{Q}$ инвариант обязан обращаться в нуль, поскольку мальцевские пополнения фундаментальных групп канонически изоморфны (см. [3]).

Отметим, что группы $\pi_{1}\left(X^{+}\right)$и $\pi_{1}\left(X^{-}\right)$на самом деле изоморфны, поскольку существует гомеоморфизм $X^{+} \rightarrow X^{-}$, индуцированный комплексным сопряжением. Но поскольку комплексное сопряжение обращает коориентацию прямых, индуцированный изоморфизм первых групп гомологий переводит канонический базис первой из них в минус канонический базис второй.

Попробуем описать автоморфизмы группы $G=\pi_{1}\left(X^{+}\right) \cong \pi_{1}\left(X^{-}\right)$.

Как мы уже отмечали, $\operatorname{Aut}\left(C_{8}\right) \cong G L_{2}\left(\mathbb{F}_{3}\right)$. Легко видеть, что подкрутка на автоморфизмы, отвечающие элементам группы $S L_{2}\left(\mathbb{F}_{3}\right)$, преобразует реализацию $\phi^{ \pm}$в проективно эквивалентную реализацию, а остальные автоморфизмы делают из $\phi^{+}$реализацию, проективно эквивалентную $\phi^{-}$, и наоборот.

Пусть $g \in G L_{2}\left(\mathbb{F}_{3}\right)$. Обозначим через $\sigma_{g}$ перестановку множества индексов $\{0,1, \ldots, 7\}$, такую, что прямая $l_{i}$ переходит в $l_{\sigma_{g}(i)}$ при соответствующем $g$ автоморфизме структуры $C_{8}$. Выберем также некоторую петлю $w_{0} \in G$, обходящую вокруг бесконечно удаленной прямой $l_{0}$ в положительном направлении. Поскольку проективная эквивалентность дает изоморфизм фундаментальных групп, мы получаем для каждого $g \in S L_{2}\left(\mathbb{F}_{3}\right)$ автоморфизм $\eta_{g}$ группы $G$, переводящий каждый из элементов $w_{i} \in G(i=0,1, \ldots, 7)$ в некоторую петлю, обходящую вокруг $l_{\sigma_{g}(i)}$ в положительном направлении, т. е. в элемент, сопряженный к $w_{\sigma_{g}(i)}$ в $G$. Аналогично, используя комплексное сопряжение, дающее изоморфизм групп $\pi_{1}\left(X^{+}\right)$и $\pi_{1}\left(X^{-}\right)$, мы получаем для $g \in G L_{2}\left(\mathbb{F}_{3}\right) \backslash S L_{2}\left(\mathbb{F}_{3}\right)$ автоморфизм $\eta_{g}$ группы $G$, переводящий каждый из элементов $w_{i} \in G(i=0,1, \ldots, 7)$ в некоторую петлю, обходящую вокруг $l_{\sigma_{g}(i)}$ в отрицательном направлении, т. е. в элемент, сопряженный к $w_{\sigma_{g}(i)}^{-1}$ в $G$.

Рассмотрим $H=G / \gamma_{2} G$. Поскольку автоморфизмы группы $G$ переводят $\gamma_{2} G$ в себя, мы можем привести любой автоморфизм $\eta \in$ Aut $G$ по модулю $\gamma_{2} G$ и получить $\bar{\eta} \in$ Aut $H$. Пусть $W=\{\bar{\eta} \mid \eta \in \operatorname{Aut} G\}$. 
Теорема 3.5. Сопоставление $g \mapsto \bar{\eta}_{g}$ является изоморфизмом групп $G L_{2}\left(\mathbb{F}_{3}\right) \rightarrow W$.

Доказательство. Определим в $H$ элемент $x_{0}=-\sum_{i=1}^{7} x_{i}$. Легко видеть, что

$$
\bar{\eta}_{g}\left(x_{i}\right)= \begin{cases}x_{\sigma_{g}(i)}, & \text { если } g \in S L_{2}\left(\mathbb{F}_{3}\right), \\ -x_{\sigma_{g}(i)}, & \text { если } g \notin S L_{2}\left(\mathbb{F}_{3}\right)\end{cases}
$$

для $i=0,1, \ldots, 7$. Отсюда видно, что сопоставление $g \mapsto \bar{\eta}_{g}$ является инъективным гомоморфизмом. Докажем сюръективность.

Пусть $\eta \in$ Aut $G$ - любой автоморфизм. Продолжим $\bar{\eta} \in$ Aut $H$ на $\Lambda^{2} H$. Очевидно, что это продолжение переводит в себя подгруппу $R_{2}=\operatorname{Ker}\left(\Lambda^{2} H \rightarrow\right.$ $P_{2}$ ), где $P_{2}=\gamma_{2} G / \gamma_{3} G$ (см. §2). Рассмотрим $R_{2}^{\perp} \subseteq \Lambda^{2} H^{*}$. Как мы знаем, абелева группа $R_{2}^{\perp}$ порождена элементами $\omega_{i j k}=\left(x_{i}^{*}-x_{j}^{*}\right) \wedge\left(x_{j}^{*}-x_{k}^{*}\right)=x_{i}^{*} \wedge x_{j}^{*}+x_{j}^{*} \wedge$ $x_{k}^{*}+x_{k}^{*} \wedge x_{i}^{*}$ при $l_{i} \succ l_{j} \cap l_{k} \in \mathscr{P}_{0}$ и $\omega_{0 i j}=\omega_{i j}=x_{i}^{*} \wedge x_{j}^{*}$ при $l_{0} \succ l_{i} \cap l_{j}$.

Лемма 3.6. Любой разложимый элемент в $R_{2}^{\perp} \subseteq \Lambda^{2} H^{*}$ для структуры $C_{8}$ пропориионален какому-то элементу вида $\omega_{i j k}$, где $p_{i j k} \in \mathscr{P}$.

Доказательство. Поскольку $H^{*}$ - свободная абелева группа, можно проводить вычисления над $\mathbb{Q}$ в $H^{*} \otimes \mathbb{Q}$. Отметим, что элементы $\omega_{i j k}$ линейно независимы и группа $\operatorname{Aut}\left(C_{8}\right)$ переводит порожденные ими одномерные подпространства в $H^{*} \otimes \mathbb{Q}$ друг в друга, действуя на множестве $\left\{\left\langle\omega_{i j k}\right\rangle \mid p_{i j k} \in \mathscr{P}\right\}$ этих подпространств транзитивно. Поэтому достаточно доказать, что если разложимый элемент $\omega$ представляется в виде линейной комбинации элементов вида $\omega_{i j k}$, причем $\omega_{012}$ входит в это разложение с коэффициентом 1 , то $\omega=\omega_{012}$.

Действительно, представим $\omega$ в виде $\omega=\sum_{0<i<j} a_{i j} x_{i}^{*} \wedge x_{j}^{*}$. Тогда $a_{12}=1$ и имеется разложение вида $\omega=\left(x_{1}^{*}+\sum_{i=3}^{7} c_{i} x_{i}^{*}\right) \wedge\left(x_{2}^{*}+\sum_{i=3}^{7} d_{i} x_{i}^{*}\right)$. С другой стороны, $\omega$ представляется в виде линейной комбинации элементов вида $\omega_{i j k}$. Сравнивая два этих разложения, легко получить, что $\omega=\omega_{012}$.

Пусть $V=H \otimes \mathbb{Q}$. Поскольку $H-$ свободный $\mathbb{Z}$-модуль, мы будем считать его вложенным в $V$. Векторное пространство $V$ можно представить как $\mathbb{Q}^{8} /\left\langle e_{0}+\cdots+e_{7}\right\rangle$, где $\left(e_{0}, \ldots, e_{7}\right)$ - стандартный базис пространства $\mathbb{Q}^{8}$. При этом $x_{i}=e_{i}+\left\langle e_{0}+\cdots+e_{7}\right\rangle$ для любого $i=0, \ldots, 7$. Легко видеть, что действие автоморфизмов структуры инцидентности $C_{8}$ на $V$ происходит из ее действия перестановками $\sigma_{g}$ базисных векторов в $\mathbb{Q}^{8}$.

Двойственное пространство $V^{*}$ представляется как подпространство в $\left(\mathbb{Q}^{8}\right)^{*}$, порожденное элементами $e_{i}^{*}-e_{j}^{*}$. Двойственный базис к базису $\left(x_{1}, \ldots, x_{7}\right)$ пространства $V$ состоит из элементов $x_{i}^{*}=e_{i}^{*}-e_{0}^{*}(i=1, \ldots, 7)$. Как мы установили, формы вида $\omega_{i j k}=\left(e_{i}^{*}-e_{j}^{*}\right) \wedge\left(e_{j}^{*}-e_{k}^{*}\right)$, где $p_{i j k} \in \mathscr{P}$, переходят под действием автоморфизма $\bar{\eta}$ в формы того же вида (с точностью до знака). Рассмотрим ядро такой формы в $V$. Оно состоит из всех векторов, в которые $e_{i}, e_{j}$ и $e_{k}$ входят с равными коэффициентами. Легко проверить, что минимальные ненулевые подпространства в $V$, получаемые пересечением ядер некоторых из $\omega_{i j k}-$ это в точности одномерные подпространства, натянутые на $x_{s}(s=0,1, \ldots, 7)$. Отсюда следует, что автоморфизм $\bar{\eta}$ переводит каждый из $x_{s}$ в $\pm x_{\sigma(s)}$ для некоторой перестановки $\sigma \in S_{8}$. Поскольку векторы $x_{s}$ связаны соотношением $\sum x_{s}=0$, все знаки «土» при $x_{\sigma(s)}$ одинаковы. Также легко проверить, представляя $\left\langle x_{s}\right\rangle$ 
как пересечение ядер форм $\omega_{i j k}$, что $\sigma=\sigma_{g}$ для некоторого $g \in G L_{2}\left(\mathbb{F}_{3}\right)$, причем знак «+» возникает при $g \in S L_{2}\left(\mathbb{F}_{3}\right)$, а «-» в противном случае. Последнее утверждение следует из того, что не существует автоморфизма $\eta: G \rightarrow G$, такого, что $\bar{\eta}\left(x_{i}\right)=-x_{i}$ для любого $i=0,1, \ldots, 7$ (иначе, применяя комплексное сопряжение, мы получили бы контрпример к теореме 3.1).

Замечание 1. Теорема 3.5 означает, по сути, что для конфигурации Маклейна стандартный базис в первой группе гомологий - абелианизации фундаментальной группы - «почти» является инвариантом самой фундаментальной группы. Более точно, инвариантом является набор циклических подгрупп, порожденных элементами стандартного базиса и элементом $x_{0}$ (циклом, охватывающим бесконечно удаленную прямую). Более того, при каждом автоморфизме набор $\left\{x_{0}, \ldots, x_{7}\right\}$ переходит либо в себя, либо в набор $\left\{-x_{0}, \ldots,-x_{7}\right\}$. Именно эта «структурная жесткость» стандартного базиса для конфигурации Маклейна будет использована в следующем параграфе.

Далеко не все конфигурации прямых обладают этим свойством. Например, для конфигурации прямых общего положения фундаментальная группа абелева, и стандартный базис первой группы гомологий ни в каком смысле не восстанавливается по фундаментальной группе ${ }^{1)}$.

\section{§4. Комбинаторно эквивалентные конфигурации с различными фундаментальными группами}

Определим структуру инцидентности $C_{13}$ как результат склеивания двух структур Маклейна следующим образом. Пусть $C_{8}^{\prime}=\left(\mathscr{L}^{\prime}, \mathscr{P}^{\prime}, \succ\right)$ и $C_{8}^{\prime \prime}=$ $\left(\mathscr{L}^{\prime \prime}, \mathscr{P}^{\prime \prime}, \succ\right)$ - два экземпляра структуры Маклейна $\left(\mathscr{L}^{\prime}=\left\{l_{0}^{\prime}, \ldots, l_{7}^{\prime}\right\}, \mathscr{P}^{\prime}=\right.$ $\left.\left\{p_{012}^{\prime}, \ldots, p_{45}^{\prime}\right\}, \mathscr{L}^{\prime \prime}=\left\{l_{0}^{\prime \prime}, \ldots, l_{7}^{\prime \prime}\right\}, \mathscr{P}^{\prime \prime}=\left\{p_{012}^{\prime \prime}, \ldots, p_{45}^{\prime \prime}\right\}\right)$. Введем отношение эквивалентности на $\mathscr{L}^{\prime} \cup \mathscr{L}^{\prime \prime}$, полагая $l_{0}^{\prime} \sim l_{0}^{\prime \prime}, l_{1}^{\prime} \sim l_{1}^{\prime \prime}, l_{2}^{\prime} \sim l_{2}^{\prime \prime}$, и отношение эквивалентности на $\mathscr{P}^{\prime} \cup \mathscr{P}^{\prime \prime}$, полагая $p_{012}^{\prime} \sim p_{012}^{\prime \prime}$. Пусть $\mathscr{P}^{\prime \prime \prime}=\left\{p_{i j}^{\prime \prime \prime} \mid i, j \in\{3, \ldots, 7\}\right\}$. Положим $\widehat{\mathscr{L}}=\left(\mathscr{L}^{\prime} \cup \mathscr{L}^{\prime \prime}\right) / \sim, \widehat{\mathscr{P}}=\left(\left(\mathscr{P}^{\prime} \cup \mathscr{P}^{\prime \prime}\right) / \sim\right) \cup \mathscr{P}^{\prime \prime \prime}$. Отношение инцидентности между $\widehat{\mathscr{L}}$ и $\left(\mathscr{P}^{\prime} \cup \mathscr{P}^{\prime \prime}\right) / \sim$ индуцируется отношениями инцидентности на $C_{8}^{\prime}$, $C_{8}^{\prime \prime}$, а отношение инцидентности между $\widehat{\mathscr{L}}$ и $\mathscr{P}^{\prime \prime \prime}$ определяется так: $p_{i j}^{\prime \prime \prime} \prec l_{i}^{\prime}, l_{j}^{\prime \prime}$. Пусть $C_{13}=(\widehat{\mathscr{L}}, \widehat{\mathscr{P}}, \succ)$. Обозначим через $\hat{l}_{i}$ класс эквивалентности прямой $l_{i}^{\prime}$ $(i=0,1,2)$ и через $\hat{p}_{012}$ класс эквивалентности точки $p_{012}^{\prime}$.

Пусть $\psi$ - проективное преобразование пространства $\mathbb{C} P^{2}$, удовлетворяющее условиям $\psi \phi^{+} l_{i}=\phi^{+} l_{i}\left(=\phi^{-} l_{i}\right)$ для $i=0,1,2$. Такое преобразование задается формулой $\left(z_{0}: z_{1}: z_{2}\right) \mapsto\left(z_{0}: z_{1}: a z_{0}+b z_{1}+c z_{2}\right)$, где $a, b, c \in \mathbb{C}, c \neq 0$. Предположим, что преобразование $\psi$ таково, что ни одна из прямых $\psi \phi^{ \pm} l_{i}, i=3, \ldots, 7$, не проходит ни через одну точку пересечения прямых $\phi^{+} l_{j}, j=0,1, \ldots, 7$. Такие $\psi$ мы будем называть общими. Построим реализации $\phi_{\psi}^{++}$и $\phi_{\psi}^{+-}$структуры $C_{13}$, склеив их из реализаций $\phi^{+}$и $\phi^{-}$структуры $C_{8}$ следующим образом: положим $\phi_{\psi}^{+ \pm} \hat{l}_{i}=\phi^{+} l_{i}$ при $i=0,1,2, \phi_{\psi}^{+ \pm} l_{i}^{\prime}=\phi^{+} l_{i}$ и $\phi_{\psi}^{+ \pm} l_{i}^{\prime \prime}=\psi \phi^{ \pm} l_{i}$ при $i=3, \ldots, 7$. Для общего преобразования $\psi$ точки пересечения прямых вида $\phi_{\psi}^{+ \pm} \hat{l}($ где $\hat{l} \in \widehat{\mathscr{L}}$ ) друг с другом находятся во взаимно однозначном соответствии с элементами из $\widehat{\mathscr{P}}$, т. е. мы получаем реализации структуры $C_{13}$. Пусть $X_{\psi}^{+ \pm}=\mathbb{C} P^{2} \backslash \bigcup \phi_{\psi}^{+ \pm} l$.

1) Я благодарен рецензенту, порекомендовавшему мне добавить это замечание. 
Отметим, что пространство общих преобразований $\psi$ связно, поэтому фундаментальные группы $G^{++}=\pi_{1}\left(X_{\psi}^{++}\right)$и $G^{+-}=\pi_{1}\left(X_{\psi}^{+-}\right)$не зависят от выбора $\psi$, с точностью до изоморфизма, согласованного с каноническим базисом в $G^{+ \pm} / \gamma_{2} G^{+ \pm}$. В связи с этим индекс $\psi$ мы можем опускать.

Теорема 4.1. Группы $G^{++} u G^{+-}$неизоморфны.

Доказательство. Пусть $\widehat{G}-$ одна из групп $G^{++}$и $G^{+-}$. Обозначим через $\hat{x}_{1}, \hat{x}_{2}, x_{3}^{\prime}, \ldots, x_{7}^{\prime}, x_{3}^{\prime \prime}, \ldots, x_{7}^{\prime \prime}$ образы стандартных образующих группы $\widehat{G}$ в $\widehat{H}=$ $\widehat{G} / \gamma_{2} \widehat{G}$. Положим $\hat{x}_{0}=-\hat{x}_{1}-\hat{x}_{2}-x_{3}^{\prime}-\cdots-x_{7}^{\prime}-x_{3}^{\prime \prime}-\cdots-x_{7}^{\prime \prime}$.

Лемма 4.2. Пусть, как в предъдущем параграфе, $G=\pi_{1}\left(X^{+}\right)$. Существуют согласованные проекции $\pi^{\prime}: \widehat{G} \rightarrow G, \pi^{\prime \prime}: \widehat{G} \rightarrow G$ и вложения $\nu^{\prime}: G \rightarrow \widehat{G}$, $\nu^{\prime \prime}: G \rightarrow \widehat{G}$, такие, что по модулю второго члена нижнего центрального ряда имеют место равенства

$$
\begin{gathered}
\bar{\pi}^{\prime}\left(\hat{x}_{i}\right)=x_{i} \quad(0 \leqslant i \leqslant 2), \quad \bar{\pi}^{\prime}\left(x_{i}^{\prime}\right)=x_{i} \quad u \quad \bar{\pi}^{\prime}\left(x_{i}^{\prime \prime}\right)=0 \quad(3 \leqslant i \leqslant 7), \\
\bar{\nu}^{\prime}\left(x_{i}\right)=\hat{x}_{i} \quad(0 \leqslant i \leqslant 2), \quad \bar{\nu}^{\prime}\left(x_{i}\right)=x_{i}^{\prime} \quad(3 \leqslant i \leqslant 7),
\end{gathered}
$$

а такљее

$$
\begin{gathered}
\bar{\pi}^{\prime \prime}\left(\hat{x}_{i}\right)=x_{i} \quad(0 \leqslant i \leqslant 2), \quad \bar{\pi}^{\prime \prime}\left(x_{i}^{\prime}\right)=0 \quad u \quad \bar{\pi}^{\prime \prime}\left(x_{i}^{\prime \prime}\right)=x_{i} \quad(3 \leqslant i \leqslant 7), \\
\bar{\nu}^{\prime \prime}\left(x_{i}\right)=\hat{x}_{i} \quad(0 \leqslant i \leqslant 2), \quad \bar{\nu}^{\prime \prime}\left(x_{i}\right)=x_{i}^{\prime \prime} \quad(3 \leqslant i \leqslant 7)
\end{gathered}
$$

в случае $\widehat{G}=G^{++} u$

$$
\begin{gathered}
\bar{\pi}^{\prime \prime}\left(\hat{x}_{i}\right)=-x_{i} \quad(0 \leqslant i \leqslant 2), \quad \bar{\pi}^{\prime \prime}\left(x_{i}^{\prime}\right)=0 \quad u \quad \bar{\pi}^{\prime \prime}\left(x_{i}^{\prime \prime}\right)=-x_{i} \quad(3 \leqslant i \leqslant 7), \\
\bar{\nu}^{\prime \prime}\left(x_{i}\right)=-\hat{x}_{i} \quad(0 \leqslant i \leqslant 2), \quad \bar{\nu}^{\prime \prime}\left(x_{i}\right)=-x_{i}^{\prime \prime} \quad(3 \leqslant i \leqslant 7)
\end{gathered}
$$

в случае $\widehat{G}=G^{+-}$.

Доказательство. Проекции строятся очевидным образом, путем выбрасывания прямых $\phi^{+} l_{i}$ при $i=3, \ldots, 7$ (или прямых $\psi \phi^{ \pm} l_{i}$ при $i=3, \ldots, 7$ ). Чтобы построить вложения, надо выбрать общее проективное преобразование $\psi$ так, чтобы прямые $\psi \phi^{ \pm} l_{i}$ при $i=3, \ldots, 7$ не пересекали некоторый открытый шар, содержащий все точки пересечения аффинных прямых $\phi^{+} l_{i}$ при $i=1, \ldots, 7$, а прямые $\phi^{+} l_{i}$ при $i=3, \ldots, 7$ не пересекали некоторый открытый шар, содержащий все точки пересечения аффинных прямых $\psi \phi^{ \pm} l_{i}$ при $i=1, \ldots, 7$. Легко видеть, что дополнения к прямым вида $\phi^{+} l_{i}$ и $\psi \phi^{ \pm} l_{i}$ в таких открытых шарах гомеоморфны соответственно пространствам $X^{+}$и $X^{ \pm}$.

Лемма 4.3. Пусть $\eta: \widehat{G} \rightarrow \widehat{G}-$ автоморфизм и $\bar{\eta}: \widehat{H} \rightarrow \widehat{H}-$ его приведение по модулю $\gamma_{2} \widehat{G}$. Тогда существуют элемент $g \in G L_{2}\left(\mathbb{F}_{3}\right), \sigma_{g}(\{0,1,2\})=$ $\{0,1,2\}$, и число $\varepsilon= \pm 1$, такие, что $\bar{\eta}\left(\hat{x}_{i}\right)=\varepsilon \hat{x}_{\sigma_{g}(i)}$ при $0 \leqslant i \leqslant 2$ и либо $\bar{\eta}\left(x_{i}^{\prime}\right)=\varepsilon x_{\sigma_{g}(i)}^{\prime} u \bar{\eta}\left(x_{i}^{\prime \prime}\right)=\varepsilon x_{\sigma_{g}(i)}^{\prime \prime}$, либо $\bar{\eta}\left(x_{i}^{\prime}\right)=\varepsilon x_{\sigma_{g}(i)}^{\prime \prime} u \bar{\eta}\left(x_{i}^{\prime}\right)=\varepsilon x_{\sigma_{g}(i)}^{\prime \prime}$ nри $3 \leqslant i \leqslant 7$. То же верно для любого изоморфизма $G^{++} \rightarrow G^{+-}$.

Доказательство следует из теоремы 3.5 и леммы 4.2 .

Лемма 4.4. Существует автоморфизм $\eta: G^{++} \rightarrow G^{++}$, такой, что $\bar{\eta}\left(\hat{x}_{i}\right)=$ $\hat{x}_{i}$ nри $0 \leqslant i \leqslant 2$ u $\bar{\eta}\left(x_{i}^{\prime}\right)=x_{i}^{\prime \prime}, \bar{\eta}\left(x_{i}^{\prime \prime}\right)=x_{i}^{\prime}$ nрu $3 \leqslant i \leqslant 7$. 
Доказательство. Поскольку для общего проективного преобразования $\psi$ нужного нам вида обратное преобразование $\psi^{-1}$ тоже является общим, $\psi$ индуцирует некоторый изоморфизм $\nu: \pi_{1}\left(X_{\psi^{-1}}^{++}\right) \rightarrow \pi_{1}\left(X_{\psi}^{++}\right)$. Рассмотрим также изоморфизм $\mu: \pi_{1}\left(X_{\psi}^{++}\right) \rightarrow \pi_{1}\left(X_{\psi^{-1}}^{++}\right)$, задаваемый гомотопией вдоль некоторого пути, соединяющего $\psi$ с $\psi^{-1}$ в пространстве общих преобразований. Поскольку изоморфизм $\mu$ согласован с каноническими базисами, а изоморфизм $\nu$ меняет местами образы прямых $l_{i}^{\prime}$ и $l_{i}^{\prime \prime}$, их композиция $\eta=\nu \mu: \pi_{1}\left(X_{\psi}^{++}\right) \rightarrow$ $\pi_{1}\left(X_{\psi}^{++}\right)$- нужный нам автоморфизм.

Лемма 4.5. Не существует автоморфизма $\eta: G^{+-} \rightarrow G^{+-}$, такого, что $\bar{\eta}\left(\hat{x}_{i}\right)=\hat{x}_{i}$ nрu $0 \leqslant i \leqslant 2$ u $\bar{\eta}\left(x_{i}^{\prime}\right)=x_{i}^{\prime \prime}, \bar{\eta}\left(x_{i}^{\prime \prime}\right)=x_{i}^{\prime}$ nрu $3 \leqslant i \leqslant 7$.

Доказательство вытекает из теоремы 3.5 и леммы 4.2 .

Утверждение теоремы легко следует из лемм 4.3, 4.4 и 4.5 .

\section{ЛитерАтУРА}

[1] W. Arvola, The fundamental group of the complement of an arrangement of complex hyperplanes, Topology, 31:4 (1992), 757-765.

[2] E. Artal Bartolo, J. Carmona Ruber, J. I. Cogolludo Agustín, M. A. Marco Buzunáriz, Invariants of combinatorial line arrangements and Rybnikov's example, in: Singularity Theory and its Applications, Adv. Studies in Pure Math., vol. 43, Math. Soc. Japan, Tokyo, 2006, 1-34.

[3] T. Kohno, On the holonomy Lie algebra and the nilpotent completion of the fundamental group of the complement of hypersurfaces, Nagoya Math. J., 92 (1983), 21-37.

[4] S. MacLane, Some interpretations of abstract linear dependence in terms of projective geometry, Amer. J. Math., 58:1 (1936), 236-241.

[5] В. Магнус, А. Каррас, Д. Солитэр, Комбинаторная теория групn, Наука, М., 1974.

[6] P. Orlik, L. Solomon, Combinatorics and topology of complements of hyperplanes, Invent. Math., 56:2 (1980), 167-189.

[7] P. Orlik, H. Terao, Arrangements of Hyperplanes, Springer-Verlag, Berlin etc., 1992.

[8] G. Rybnikov, On the fundamental group of the complement of a complex hyperplane arrangement, DIMACS Tech. Report 94-13 (1994), 33-50; http://arxiv.org/ abs/math/9805056.

[9] D. J. A. Welsh, Matroid Theory, Academic Press, London-New York, 1976.

[10] G. M. Ziegler, Matroid representations and free arrangements, Trans. Amer. Math. Soc., 320:2 (1990), 525-541.

Высшая школа экономики, факультет математики e-mails: gr@mccme.ru, grybnikov@hse.ru

Поступило в редакцию 11 января 2010 г. 